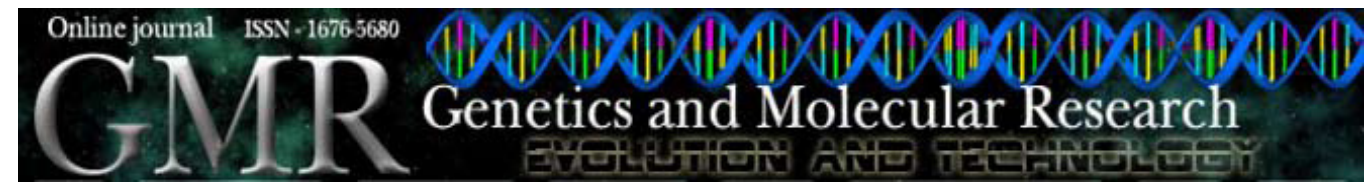

\title{
Utility of STR markers for the molecular diagnosis of a large Brazilian family with Charcot-Marie-Tooth disease
}

\author{
C.O. Possamai ${ }^{1}$, F.M. Carvalho ${ }^{1}$, M.F.C. Silva ${ }^{1}$, E.V. Wolfgramm ${ }^{1}$, \\ M.P.N. Sartori ${ }^{1}$, F.S.V. Malta ${ }^{2}$, V.P. Ribeiro ${ }^{2}$, V.P. Spina ${ }^{2}$, \\ K.B. Gomes ${ }^{2}$, A.C.S. Ferreira ${ }^{2}$ and I.D. Louro ${ }^{1}$ \\ ${ }^{1}$ Departamento de Ciências Biológicas, \\ Centro de Ciências Humanas e Naturais, \\ Núcleo de Genética Humana e Molecular, \\ Universidade Federal do Espírito Santo, Vitória, ES, Brasil \\ ${ }^{2}$ Departamento de Genética Humana, \\ Instituto Hermes Pardini, Belo Horizonte, MG, Brasil \\ Corresponding author: I.D. Louro \\ E-mail: iurilouro@yahoo.com
}

Genet. Mol. Res. 7 (4): 1179-1185 (2008)

Received July 28, 2008

Accepted August 21, 2008

Published October 28, 2008

\begin{abstract}
Charcot-Marie-Tooth type 1A disease (CMT1A) is most frequently caused by a tandem DNA duplication of a 1.4-Mb genomic fragment in the 17p11.2-12 chromosomal region. The disease is probably the product of a dosage effect of the peripheral myelin protein 22 gene located within the duplicated segment. We sought to study the largest reported Brazilian family with suspected diagnosis of CMT1A using eight short tandem repeat microsatellite markers. In addition, we analyzed the informativeness of these markers in the normal Brazilian population. The duplication was found in 12 members of the family. In two patients with CMT1A symptoms, the duplication was not detected, and one asymptomatic subject showed the duplication. D17S2230, D17S9B, D17S2220, D17S2227, D17S9A, and D17S4A markers showed the highest heterozygosity rates, and D17S2228 and
\end{abstract}


D17S2224 markers were the least informative in our analysis.

Key words: Charcot-Marie-Tooth 1A; 17p11.2-p12 duplication; Short tandem repeat markers; Molecular diagnosis; Brazilian population

\section{INTRODUCTION}

Charcot-Marie-Tooth (CMT) disease is a heterogeneous group of hereditary peripheral neuropathies, occurring with an estimated prevalence of 1 in 2500 (Skre, 1974). Two major clinical forms have been identified, type 1 (CMT1) and type 2 (CMT2). CMT1A is the most common form, characterized by pes cavus deformity of the feet, reduced or absent deep tendon reflexes, slowly progressive distal muscle atrophy and weakness, demyelination of nerve fibers, and reduced nerve conduction velocities in both motor and sensory neurons (Lupski and Garcia, 1992). A dominantly inherited $1.4-\mathrm{Mb}$ duplication at the $17 \mathrm{p} 11.2-12$ region is responsible for most $\mathrm{CM}$ T1A cases, probably arising after unequal crossing-over between repeated sequences flanking this region (Figure 1) (Lupski et al., 1991; Wise et al., 1993). The gene encoding peripheral myelin protein 22 (PMP22) maps in the middle of this 1.4-Mb genomic fragment, and several lines of evidence indicate that alterations in gene dosage of PMP22 (three copies) are responsible for the pathogenesis of CMT1A, whereas loss of one copy of PMP22 results in a clinically distinct hereditary neuropathy with liability to pressure palsy (Badano et al., 2001; Inoue et al., 2001).

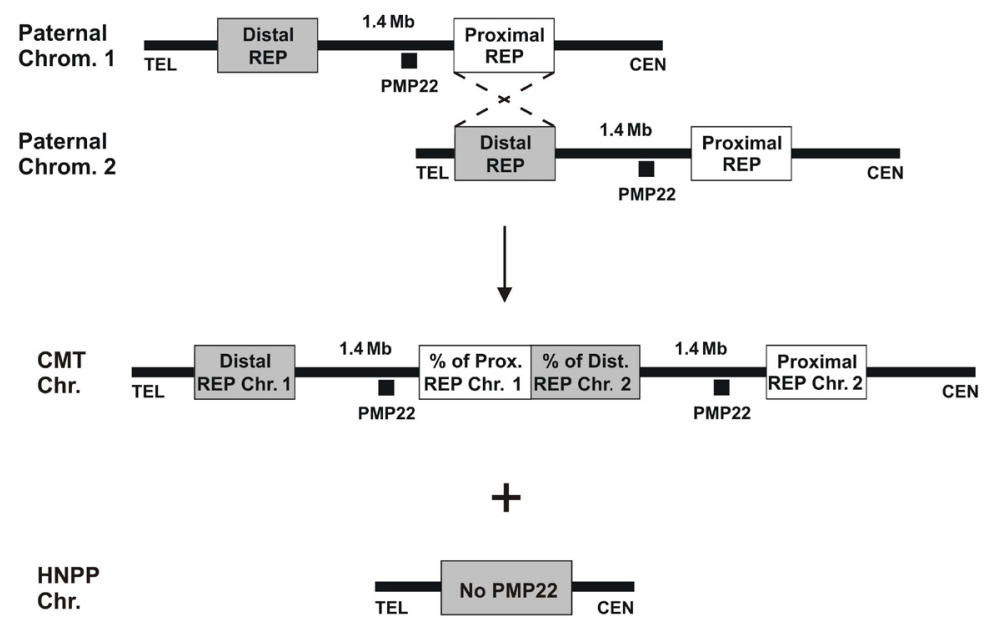

Figure 1. Schematic mechanism for unequal crossing-over. Recombination between misaligned flanking repeats (REP) of chromosome 17p11.2-12, flanking the peripheral myelin protein 22 gene (PMP22), during meiosis lead to a duplication of the gene (offspring with three copies of PMP22) causing Charcot-Marie-Tooth type 1A disease (CMT1A) and a deletion (offspring with one copy of PMP22), causing hereditary neuropathy with liability to pressure palsies $(\mathrm{HNPP})$. $\mathrm{CEN}=$ centromere; $\mathrm{TEL}=$ telomere; $\mathrm{Chr} .=$ chromosome.

Several methods for the molecular diagnosis of CMT1A duplications have been developed (Choi et al., 2006). Polymerase chain reaction (PCR)-based methods using short tandem repeats (STRs) that detect three different alleles in CMT1A duplications in combination with semiquantitative dosage measurements have been widely used, mainly because of their cost effectiveness and speed. In the present study, we validated several STR markers for usage in the Brazilian 
population, determining allele frequencies in normal individuals, as well as heterozygosity rates, followed by an analysis of a large Brazilian family with clinical symptoms of CMT1A. Our results suggest that the duplication was caused by an unequal crossing-over between paternal and maternal chromosomes, using repetitive sequences that flank the duplicated segment.

\section{SUBJECTS AND METHODS}

\section{Subjects}

The main sample in this study was a four-generation family from the northwest region of Espírito Santo State, Brazil. Many individuals of this family showed clinical features of the CMT1A disease, characterizing the largest Brazilian family with this condition so far reported.

An additional 100 samples from unrelated healthy Brazilian individuals, from all regions of the country, were included in the study in order to calculate allele frequencies and heterozygosity rates of the general Brazilian population. Informed consent was obtained from all participants. DNA was extracted from peripheral blood leukocytes using the Gentra Puregene commercial kit (Qiagen, CA, USA).

This study was approved by the Research Ethics Committee of the Universidade Federal do Espírito Santo (protocol No. CEP-036/06).

\section{Identification of the CMT1A duplication}

The chromosome 17p11.2-p12 duplication was determined using three fluorescent multiplex systems, containing the following eight microsatellite markers mapping within the CMT1A duplicated region: D17S2220, D17S2230, D17S2228, D17S9B, D17S2227, D17S2224, D17S9A, and D17S4A (Figure 2). PCR was performed in three multiplex mixes: mix one (D17S2220, D17S2230, D17S2228, and D17S9B), mix two (D17S2224 and D17S2227) and mix three (D17S9A and D17S4A) using FAM- or HEX-labeled primers described previously (Badano et al., 2001; Latour et al., 2001). Amplification was carried out in a Gene Amp PCR system 2400 (PE, Applied Biosystems), using amplification conditions described previously (Badano et al., 2001). The CMT1A duplication was determined by visualization of three fragments (STR alleles) after electrophoretic separation or two fragments with a 2:1 fluorescence intensity ratio, using a Hitachi FMBIO $^{\circledR}$ II Fluorescence Imaging System.

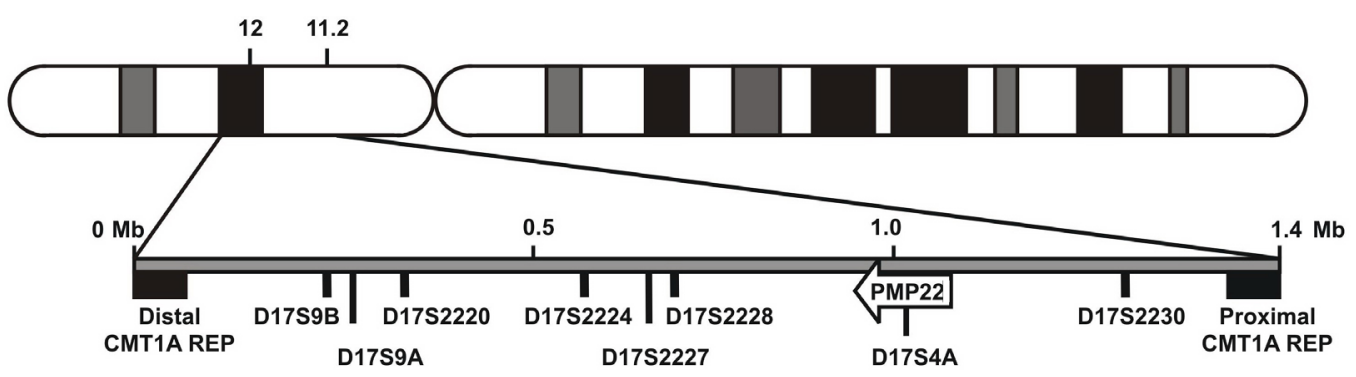

Figure 2. Chromosomal localization of short tandem repeat (STR) markers. 17p11.2-p12 STR markers between proximal and distal Charcot-Marie-Tooth type 1A disease (CMT1A) flanking repeat sequences (REP). The D17S4A STR marker is located within the peripheral myelin protein 22 (PMP22) gene. 


\section{Allelic frequency calculation}

Allele frequencies were estimated using the PowerStats, version 12, an Excel spreadsheet template developed for population genetic calculations (Jones, 1972; Botstein et al., 1980; Brenner and Morris, 1989).

\section{RESULTS}

\section{Family pedigree}

The family studied was composed of 42 individuals (four generations), of whom 16 showed clinical symptoms of CMT1A. Three of these were not available for testing (I-1 was deceased, II-4 and IV-2 were inaccessible). Twenty-one normal individuals were not tested for the reasons above, as well as for not complying with the study. Figure 3 shows the family pedigree.

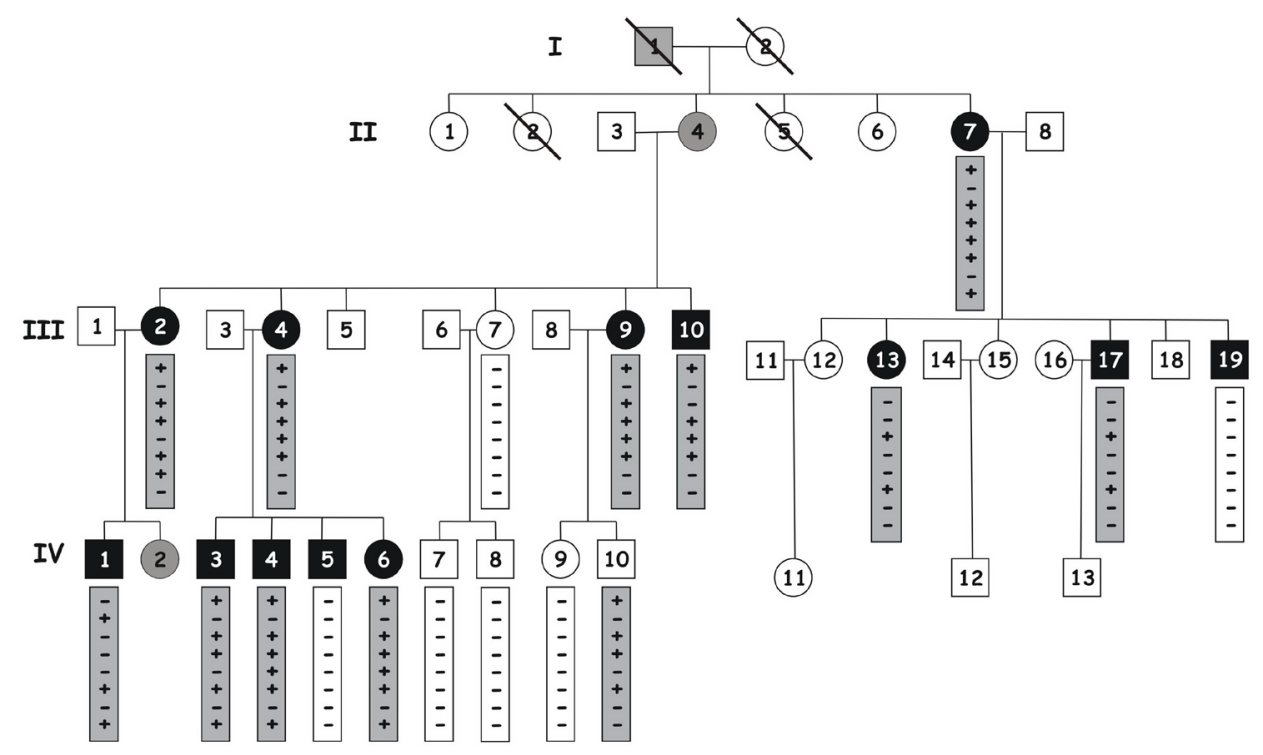

Figure 3. Family pedigree. Open symbols represent asymptomatic individuals, black symbols represent symptomatic individuals, and gray symbols represent untested individuals with positive history. Presence of three different alleles is marked with a plus $(+)$ and presence of one or two different alleles is marked with a minus (-). Gray boxes confer a positive molecular diagnosis for the individual. Short tandem repeat markers represented inside the boxes are, from top to bottom: D17S2227, D17S2224, D17S2220, D17S9A, D17S4A, D17S2230, D17S2228, and D17S9B.

\section{Molecular diagnosis of the CMT1A duplication}

Blood samples from 18 family members were analyzed and 12 duplications were found. A panel of results showing STR markers and family members is shown in Figure 3. Results were based on the number of different alleles visualized on the gel and fluorescence intensity ratio. 
The pedigree strongly suggests that the affected individual I-1 transmitted the defective chromosome containing the duplication to subsequent generations. In two individuals with CMT symptoms (III-19 and IV-5) the duplication was not detected. One asymptomatic individual (IV-10) had the duplication, but showed no symptoms probably because he was still a child (Garcia et al., 1998).

\section{Allele frequencies}

Allele frequencies and heterozygosity rates for the normal Brazilian population were estimated by testing unaffected and unrelated controls collected throughout the country. Calculations were performed by the PowerStats spreadsheet (Jones, 1972; Botstein et al., 1980; Brenner and Morris, 1989). Altogether, 30 alleles were observed for eight STR markers (Table 1). Eighteen alleles were observed for D17S2230, which was the most polymorphic marker, and five alleles for D17S2228, the least polymorphic one. D17S2230 showed the highest heterozygosity rate (0.91), followed by D17S9B and D17S2220 with heterozygosity rates of 0.84 and 0.82 , respectively. D17S2228 and D17S2224 markers showed the lowest heterozygosity rates ( 0.44 and 0.61 , respectively).

\begin{tabular}{|c|c|c|c|c|c|c|c|c|}
\hline Allele & D17S4A & D17S9A & D17S9B & $\mathrm{D} 17 \mathrm{~S} 2220$ & D17S2224 & D17S2227 & D17S2228 & D17S2230 \\
\hline 3 & 42.7 & - & - & - & - & - & - & - \\
\hline 4 & 5.1 & - & - & - & - & - & - & - \\
\hline 5 & - & - & - & - & 2.7 & - & - & - \\
\hline 6 & 12.4 & - & - & - & 13.2 & - & - & - \\
\hline 7 & - & - & - & - & 39.6 & - & - & - \\
\hline 8 & 35.4 & - & 1.1 & - & 32.4 & - & - & - \\
\hline 9 & 1.1 & - & 0.6 & - & 9.3 & 1.1 & - & - \\
\hline 10 & 2.2 & 2.2 & 3.3 & - & 0.5 & 2.2 & - & - \\
\hline 11 & 1.1 & 5.0 & 1.7 & - & 1.6 & 5.1 & - & 1.1 \\
\hline 12 & - & 7.8 & 15.6 & - & 0.5 & 15.2 & 4.5 & 2.2 \\
\hline 13 & - & 24.4 & 27.2 & - & - & 23.6 & 53.4 & 4.5 \\
\hline 14 & - & 30.0 & 24.4 & - & - & 25.3 & 24.2 & 6.2 \\
\hline 15 & - & 18.9 & 15.6 & - & - & 15.7 & 16.9 & 7.9 \\
\hline 16 & - & 9.4 & 7.2 & - & - & 10.7 & - & 15.2 \\
\hline 17 & - & 2.2 & 2.8 & 2.8 & - & 1.1 & 1.1 & 10.1 \\
\hline 18 & - & - & 0.6 & 1.7 & - & - & - & 9.6 \\
\hline 19 & - & - & - & 3.4 & - & - & - & 7.9 \\
\hline 20 & - & - & - & 5.1 & - & - & - & 7.3 \\
\hline 21 & - & - & - & 11.2 & - & - & - & 9.0 \\
\hline 22 & - & - & - & 18.0 & - & - & - & 3.4 \\
\hline 23 & - & - & - & 14.6 & - & - & - & 1.7 \\
\hline 24 & - & - & - & 12.4 & - & - & - & 2.2 \\
\hline 25 & - & - & - & 12.4 & - & - & - & 5.1 \\
\hline 26 & - & - & - & 5.1 & - & - & - & 2.8 \\
\hline 27 & - & - & - & 3.4 & - & - & - & 1.7 \\
\hline 28 & - & - & - & 6.2 & - & - & - & 2.2 \\
\hline 29 & - & - & - & 2.2 & - & - & - & - \\
\hline 30 & - & - & - & 0.6 & - & - & - & - \\
\hline 31 & - & - & - & 0.6 & - & - & - & - \\
\hline 32 & - & - & - & 0.6 & - & - & - & - \\
\hline $\mathrm{H}_{\mathrm{obs}}$ & 0.73 & 0.77 & 0.84 & 0.82 & 0.61 & 0.79 & 0.44 & 0.91 \\
\hline
\end{tabular}


For diagnostic purposes, marker D17S2230 was the most informative, showing three different alleles in all 12 subjects with the duplication. Markers D17S2220, D17S2227 and D17S9A were also very informative, featuring three different alleles in ten, nine and nine subjects, respectively. In contrast, markers D17S2224 and D17S2228 were informative for only one patient.

\section{DISCUSSION AND CONCLUSION}

To our knowledge, this is the largest Brazilian family with the CMT1A disease that has been described. We observed an autosomal dominant inheritance pattern in this family, in which the oldest affected ancestral (I-1) appears to have directly or indirectly transmitted the defective chromosome to at least 12 members of the family (Figure 3).

The duplication was found in 11 of 13 symptomatic subjects. The two members of the family with clinical signs of CMT1A and without detectable CMT1A duplication probably inherited alleles from the non-affected parent that were also present in the duplication, in such a way that three different alleles were never found for the STR markers used. A 2:1 fluorescence ratio should be diagnostic in these cases; however, it is a quantitative method, and not as clear as finding three different alleles for one or more markers. Further investigation with more markers should ultimately find markers with three alleles in these individuals. We do not believe that these two patients had a different disease with similar symptoms or a different gene causing CMT, simply because they were part of a large CMT family with a detected duplication in most affected individuals.

Garcia et al. (1998) described children with quite subtle CMT1A clinical signs, and they also observed that in 13 CMT1A pedigrees, three adult carriers had no signs of disease. Nicholson (1991) reported that two of 75 CMT1A patients, aged seven and ten years, had a normal examination. In some CMT1A patients, exuberant symptoms do not occur until the second decade of life. This could be the reason for the lack of symptoms in individual IV-10, in whom the duplication was detected.

Allelic frequency analysis showed locus D17S2230 as the most polymorphic one, a result also found in other studies (Badano et al., 2001; Choi et al., 2006). D17S2230, D17S9B, D17S2220, D17S2227, D17S9A, and D17S4A markers showed the highest heterozygosity rates in normal controls. With the exception of D17S9B and D17S4A, these markers were also very informative for the family studied. D17S2228 and D17S2224 markers were the least informative ones for both normal controls and affected individuals of the family studied. We suggest that these two markers be excluded from the panel used for the Brazilian population, and carefully reconsidered for other specific populations around the world.

\section{ACKNOWLEDGMENTS}

C.O. Possamai and F.M. Carvalho were recipients of CNPq scholarships.

\section{REFERENCES}

\footnotetext{
Badano JL, Inoue K, Katsanis N and Lupski JR (2001). New polymorphic short tandem repeats for PCR-based CharcotMarie-Tooth disease type 1A duplication diagnosis. Clin. Chem. 47: 838-843.

Botstein D, White RL, Skolnick M and Davis RW (1980). Construction of a genetic linkage map in man using restriction fragment length polymorphisms. Am. J. Hum. Genet. 32: 314-331.
} 
Brenner C and Morris J (1989). Paternity index calculations in single locus hypervariable DNA probes: validation and other studies. In: Proceedings for the International Symposium on Human Identification Promega Corporation, Madison, 21-53.

Choi BO, Kim J, Lee KL, Yu JS, et al. (2006). Rapid diagnosis of CMT1A duplications and HNPP deletions by multiplex microsatellite PCR. Mol. Cell 23: 39-48.

Garcia A, Combarros O, Calleja J and Berciano J (1998). Charcot-Marie-Tooth disease type 1A with 17p duplication in infancy and early childhood: a longitudinal clinical and electrophysiologic study. Neurology 50: 1061-1067.

Inoue K, Dewar K, Katsanis N, Reiter LT, et al. (2001). The 1.4-Mb CMT1A duplication/HNPP deletion genomic region reveals unique genome architectural features and provides insights into the recent evolution of new genes. Genome Res. 11: 1018-1033.

Jones DA (1972). Blood samples: Probability of discrimination. J. Forensic Sci. Soc. 12: 355-359.

Latour P, Boutrand L, Levy N, Bernard R, et al. (2001). Polymorphic short tandem repeats for diagnosis of the CharcotMarie-Tooth 1A duplication. Clin. Chem. 47: 829-837.

Lupski JR and Garcia CA (1992). Molecular genetics and neuropathology of Charcot-Marie-Tooth disease type 1A. Brain Pathol. 2: 337-349.

Lupski JR, de Oca-Luna RM, Slaugenhaupt S, Pentao L, et al. (1991). DNA duplication associated with Charcot-MarieTooth disease type 1A. Cell 66: 219-232.

Nicholson GA (1991). Penetrance of the hereditary motor and sensory neuropathy Ia mutation: assessment by nerve conduction studies. Neurology 41: 547-552.

Skre H (1974). Genetic and clinical aspects of Charcot-Marie-Tooth's disease. Clin. Genet. 6: 98-118.

Wise CA, Garcia CA, Davis SN, Heju Z, et al. (1993). Molecular analyses of unrelated Charcot-Marie-Tooth (CMT) disease patients suggest a high frequency of the CMTIA duplication. Am. J. Hum. Genet. 53: 853-863. 